\title{
Pollution De L'eau De Consommation Humaine Et Risques Sanitaires A Court Terme : Cas Du Bassin Versant De La Menoua (Ouest-Cameroun)
}

\author{
Santsa Nguefack Charles Vital \\ Ndjouenkeu Robert \\ Département de Sciences Alimentaires et Nutrition, ENSAI, \\ Université de Ngaoundéré, Cameroun \\ Ngassoum Martin Benoît \\ Département Chimie Appliquée, ENSAI, \\ Université de Ngaoundéré, Cameroun
}

Doi: 10.19044/esj.2018.v14n3p96 URL:http://dx.doi.org/10.19044/esj.2018.v14n3p96

\begin{abstract}
The health risk associated with the consumption of water polluted by humans in the World, particularly in Africa, is a real public health problem. This is why WHO, from its inception in 1948, has always been concerned with defining limit values. The aim of this work was to study the physicochemical and microbiological quality of water in the Menoua watershed through a spatiotemporal dynamics to assess its degree of anthropogenic pollution and to estimate the level of risk of infection and diseases inherent in its consumption. Physicochemical analysis $(\mathrm{pH}$, temperature, turbidity and dissolved oxygen) and microbiological (E-coli, salmonella and shigellas) was carried out on 132 samples taken from 33 sites distributed in two seasons, two in dry seasons and two in rainy season. The results show that these waters have an acidic $\mathrm{pH}$, a low dissolved oxygen content, strong microorganism levels all correlated with high turbidity. These very high levels of microorganism, illustrate well the effect of the action of the man on the pollution of the water. This pollution could have several origins, the most important of which are related to spilled household discharges, without any prior treatment in rivers, manure from livestock and used in agriculture. The level of microbiological risk is very high for consumers and typhoid is the most recurrent waterborne disease in the region. This diagnosis would help decision-makers to better pronounce on the management of this resource in order to reduce the health risks inherent in its consumption.
\end{abstract}

Keywords: Health risk, pollution, microorganism, la Menoua catchment, physicochemical, anthropogenic activities, waterborne disease 


\section{Résumé}

Le risque sanitaire lié à la consommation de l'eau polluée par l'homme dans le Monde plus particulièrement en Afrique, est une véritable problématique en matière de santé publique. C'est pourquoi l'OMS, dès sa création en 1948, s'est toujours préoccupée de définir les valeurs limites. L'objectif de ce travail était d'étudier le qualité physicochimique et microbiologique de l'eau du bassin versant de la Menoua à travers une dynamique spatiotemporelle pour évaluer son degré de pollution anthropique et d'estimer le niveau de risque d'infection et des maladies inhérentes à sa consommation. L'analyse physicochimique $(\mathrm{pH}$, température, turbidité et Oxygène dissous) et microbiologique (E-colis, salmonelles et shigellas) a été faite sur 132 échantillons prélevés dans 33 sites répartis en deux saisons dont deux en saisons sèche et deux autres en saison pluvieuse. Il ressort des résultats que ces eaux ont un $\mathrm{pH}$ acide, une faible teneur en Oxygène dissous, des fortes teneurs en microorganisme le tout corrélé à une forte turbidité. Ces teneurs très élevées en microorganisme, illustrent bien l'effet de l'action de l'homme sur la pollution de l'eau. Cette pollution pourrait avoir plusieurs origines dont les plus importantes sont liées aux rejets ménagers déversés, sans aucun traitement préalable dans les cours d'eau, aux fumiers provenant de l'élevage et utilisés en agriculture. Le niveau de risque microbiologique est très élevé pour les consommateurs et la typhoïde est la maladie hydrique la plus récurrente dans la région. Dans le cadre de cette perspective partipative ce diagnostic aiderait les décideurs à mieux se prononcer sur la gestion de cette ressource en vue de diminuer les risques sanitaires inhérents à sa consommation.

Mots-clés: risque sanitaire, pollution, microorganisme, bassin versant de la Menoua, physicochimique, activités anthropiques, maladie hydrique

\section{Introduction}

L'eau constitue un élément indispensable pour la vie de l'homme. Vue sur cet angle, elle mérite une attention toute particulière, vu qu' elle se trouve très dégradée et sérieusement menacée par les activités anthropiques. En effet, la croissance démographique toujours galopante accompagnée d'une urbanisation rapide et anarchique et les progrès réalisés en termes d'industrialisation qui causent de nombreuses perturbations pour les milieux naturels (Mc Kinney, 2002), l'intensification des activités agropastorales impliquant ainsi l'utilisation abusive et non contrôlées des intrants (engrais et phytosanitaires) et le manque d'éducation de la population envers la protection de cette ressource, conduisent à générer des éléments polluants qui peuvent modifier les paramètres de la qualité de l'eau (Nkhuwa, 2003 ; Hassoune et al., 2006). Par ailleurs, la pollution de ces eaux représente un aspect très 
inquiétant, d'autant plus que leurs utilisations à des fins alimentaires constituent des risques sanitaires chez les consommateurs (Laferriere et al., 1996 ; El Kettani et al., 2006). Cette problématique est plus sérieuse dans certains pays d'Afrique, notamment au Cameroun, où certaines régions connaissent une impressionnante poussée démographique et une intensification des activités agropastorales. En effet, 90\% des rejets domestiques ne sont pas traités et polluent les ressources en eau disponibles (Kamgho, 2010) et est par conséquent, à l'origine des maladies liées à l'eau telles que: la conjonctivite, la méningite, les maladies diarrhéiques, la typhoïde, la dysenterie amibienne (Duteurtre et al., 2004). Selon PLAN Cameroun, les enfants en bas âge et les femmes demeurent les couches les plus vulnérables face à ce problème, qui ne laisse pas en marge le département de la Menoua (Kengni et al., 2012).

Dschang, chef-lieu de ce département, région de l'ouest Cameroun, subit une croissance spatiale et démographique rapide et un développement anarchique de l'habitat (ONU Habitat, 2005). Son statut de ville universitaire influence énormément la poussée démographique. On estime la population à environ 182,3 habitants au $\mathrm{km}^{2}$ avec un taux de croissance de 4,7\% par an (INS, 2010). Cette région est alimentée en eau par le bassin versant de la Menoua qui souffre d'un grand problème de pollution induite par des activités anthropiques très développées dans la région dont les origines pourraient être liées aux engrais et aux phytosanitaires utilisés par les agriculteurs et les éleveurs, en vue d'améliorer leurs productions d'une part, et d'autre part, à la proximité de certains points de décharges ou de l'écoulement des eaux usées dans les cours d'eau du bassin ou encore leurs infiltrations dans le sol (Fonteh et al., 2013). Les risques que peuvent apporter de telles actions ne seront considérés que néfastes sur le plan sanitaire. Des études similaires ont été réalisées dans d'autres régions du Cameroun notamment Yaoundé, Douala, Bafoussam, Mokolo: (Djeuda et al.,1998; ERA-Cameroun, 2001; Wethé et al., 2003; Kuitcha et al., 2008; Mpakam et al., 2009; Kouam et al., 2011; Ngnikam et al., 201; Lewa et al.,2014).

Le présent travail vise à étudier la qualité physicochimique et microbiologique de l'eau du bassin versant de la Menoua à travers une dynamique spatiotemporelle pour évaluer le degré de pollution provenant des activités anthropiques et l'estimation du niveau de risque d'infection et des maladies inhérentes à sa consommation. La démarche du travail s'est appuyée sur deux hypothèses: La première, selon laquelle le degré de pollution de l'eau du bassin versant de la Menoua varie dans l'espace et le temps, et la deuxième selon laquelle le niveau de risque d'infection et l'endémicité des pathologies augmentent avec le degré de contamination de l'eau que consomment les populations. 


\section{Matériel et méthode \\ Présentation du site}

Dschang, chef-lieu du département de la Menoua, région de l'Ouest Cameroun, subit une croissance spatiale et démographiques rapides et un développement anarchique de l'habitat (ONU Habitat, 2005). Son statut de ville universitaire influence énormément la poussée démographique. On estime la population à environ 182,3 habitants au $\mathrm{km}^{2}$ avec un taux de croissance de 4,7\% par an (INS, 2010). La ville de Dschang est comprise entre $5^{\circ} 25^{\prime}-5^{\circ} 30^{\prime}$ de Latitude Nord et $10^{\circ} 10^{\circ} 5^{\prime}$ de Longitude Est et est située à $213 \mathrm{~km}$ au nord de Douala et $350 \mathrm{~km}$ au nord-ouest de Yaoundé. Elle est bâtie sur le versant Sud-Est des Monts Bamboutos et s'ouvre au Sud-Ouest par la rivière Menoua qui coule vers la plaine des Mbo. A l'Est, elle est fermée par le massif du Bani qui culmine à plus de $1920 \mathrm{~m}$. La moyenne des précipitations est de $1900 \mathrm{~mm} /$ an pour une moyenne de température de $20,2{ }^{\circ} \mathrm{C}$ (minima de $\left.13,4^{\circ} \mathrm{C}\right)$ (Da Costa, 2004). Dschang est comprise entre la savane d'altitude et la forêt montagnarde; Les bas-fonds sont recouverts d'une végétation très dense. Le relief est très accidenté; la ville repose sur un socle ancien recouvert de formations volcaniques. La zone urbaine occupe essentiellement un ensemble de collines. Les fonds des vallées sont occupés par des marigots et des terrains marécageux. La ville est soumise au régime climatique d'altitude (type camerounien). Cette ville est alimentée en eau par le bassin versant de la Menoua (Figure1). Les activités anthropiques les plus rencontrées dans cette localité sont l'agriculture et l'élevage.

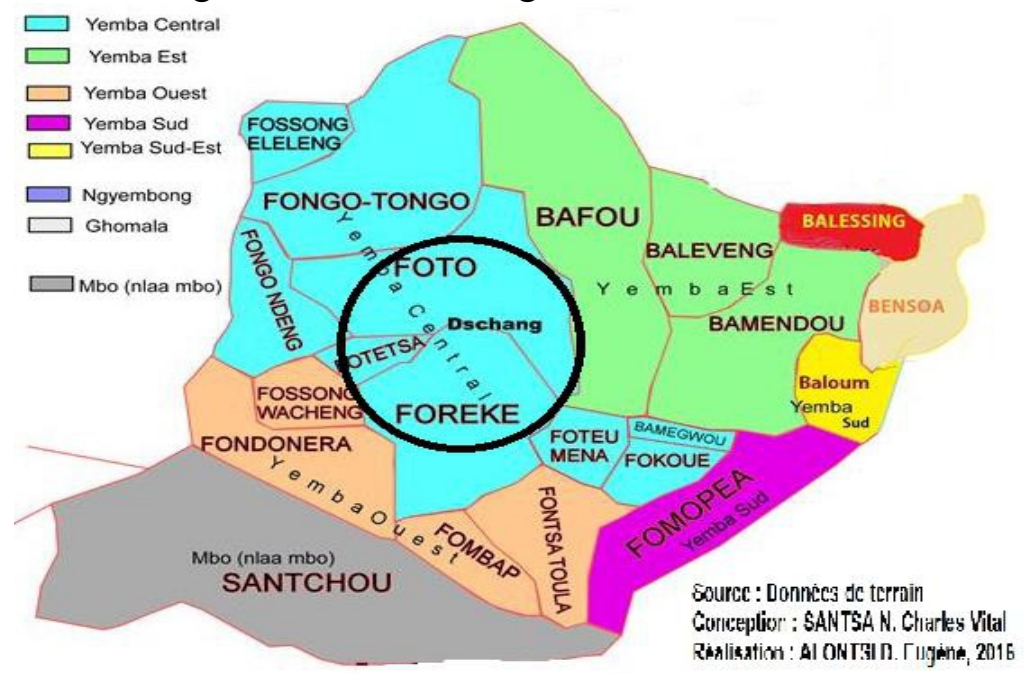

Figure 1: Carte du milieu d'étude

\section{Sites d'étude}

Pour la réalisation de ce travail, le choix des sites de prélèvement des différents échantillons d'eau s'est fait en tenant compte des diverses activités 
anthropiques recensées: e= élevage, a= agriculture, $\mathrm{d}=$ décharge des déchets, $\mathrm{b}=$ bâti. Les coordonnées géographiques de chaque site ont été prises en compte à l'aide de l'appareil GPS. A cet effet, 33 sites ont été choisis et repartis comme suit: Eau de source 15 sites; Eau de forage 7 sites; Eau de puits 11 sites. Ainsi, une carte de localisation des sites d'étude a été faite avec le logiciel Quantum GIS (Figure 2). Quatre (4) campagnes de prélèvements ont été faites dans les 33 sites dont deux en saisons sèche (décembre et février 2014/2015) et les autres en saison pluvieuse (mai et août 2015).

\section{Dispositif, prélèvement et transport des différents échantillons d'eau.}

Les flacons stériles de $500 \mathrm{ml}$ ont été étiquetés et les prélèvements des échantillons ont été effectués et conservés dans une enceinte réfrigérée à $4{ }^{\circ} \mathrm{C}$ suivant les procédures décrites par Rodier et al. (1996).

\section{Mesure des paramètres physico-chimiques de l'eau}

Sur le terrain, la Température a été relevée à l'aide d'un thermomètre électronique de marque Digital Thermometer, les mesures du pH ont été faites à l'aide d'un pH-mètre de marque SUNTEX TS-2, les mesures de la turbidité (exprimées en FTU) ont été effectuées à l'aide du multimètre conductivity/TDS de marque HACH, Oxygène dissous (exprimé en mg/l) a été mesuré à l'aide d'un oxymètre ORION Research, l'analyser modèle 607 A avec électrode spécifique.

\section{Mesure des paramètres microbiologiques de l'eau}

La caractérisation microbiologique a été portée sur les teneurs en indicateurs de contamination fécale décrite par (Durand et Lévêque, 1980; APHA et al., 1981; Rodier, 1996). Les teneurs en indicateurs de contamination fécale etait axées sur la détermination du taux d'E-coli, de salmonelle et shigella à l'aide de la technique des membranes filtrantes. Les milieux de culture bactérienne ont été préparés pour ces différents organismes suivant les prescriptions du manufacturier et des manuels d'analyse de l'eau (APHA et al., 1981; Sloat et Ziel, 1992; Rodier, 1996) et stérilisés à $121^{\circ} \mathrm{C}$ pendant 15 minutes à l'autoclave. Ces milieux liquides ont été répartis dans les boîtes de Pétri et se solidifient en refroidissant.

Les boîtes de Pétri contenant les échantillons d'eau destinés à la culture des E-colis, Salmonelles et shigellas ont été incubés à conditions aérobie pendant 24 à $48 \mathrm{~h}$, entre 35 et $37^{\circ} \mathrm{C}$ dans des incubateurs: pour Ecolis, les points de croissance des colonies étaient de couleur rose à rouge, (colonies parfois cernées par une zone de précipitation biliaire); pour salmonelles, les points de croissance des colonies étaient de couleur beige; pour les shigellas, les points de croissance étaient de couleur beige. Tous ces éléments ont été identifiés sur les différents filtres et comptés. La 
détermination de la concentration de ces dernières dans les divers échantillons a été faite à l'aide d'un compteur et exprimée en UFC/100ml.

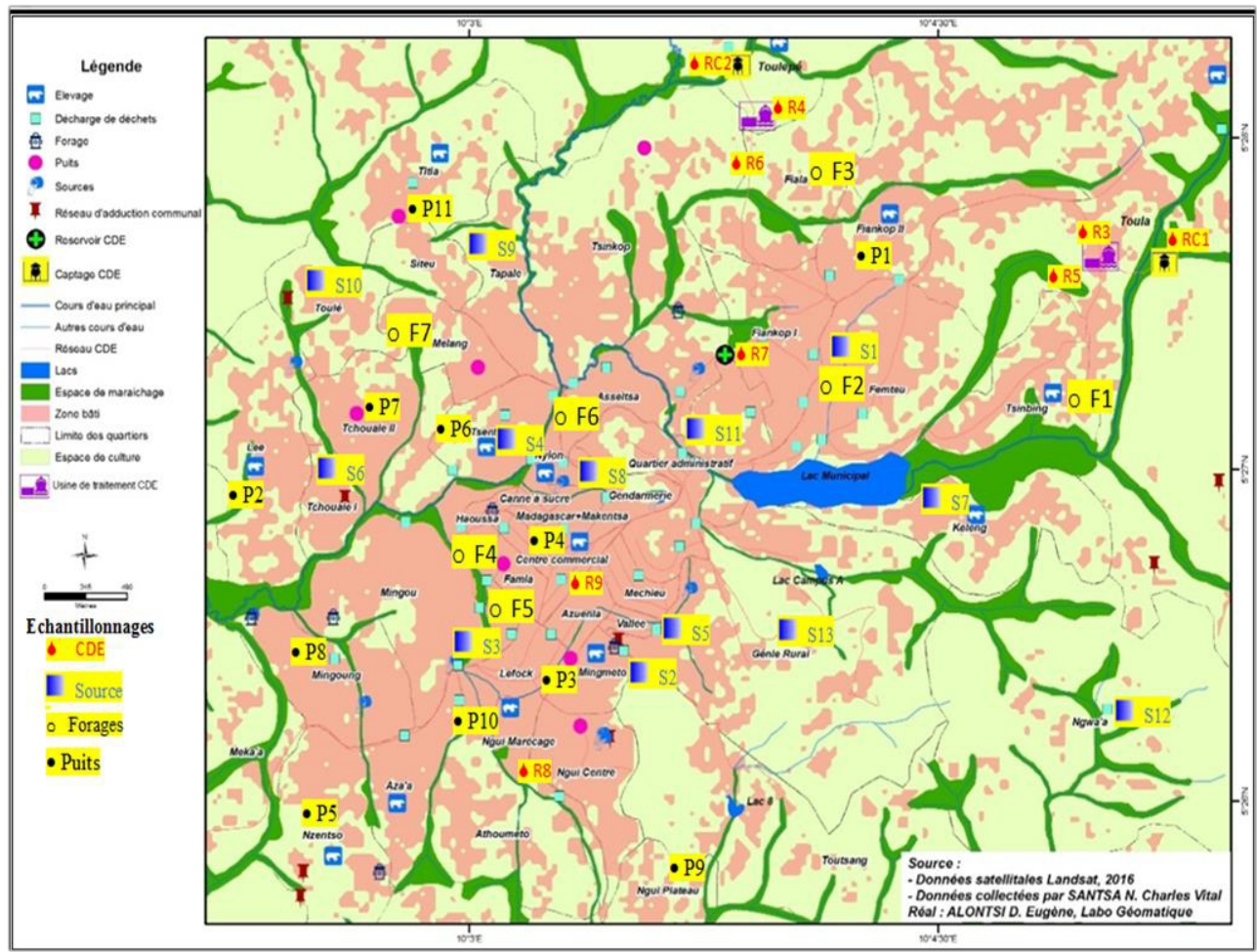

Figure 2: Carte des sites d'échantillonnage

\section{Evaluations quantitatives du niveau de risque microbiologique(EQRM)}

C'est une méthode qui permet d'estimer par calcul les risques d'origine anthropique ou naturelle pour la santé et auxquels est soumise une population donnée. L'EQRM est calquée sur la démarche générale de l'évaluation des risques chimiques (EDR) et permet alors de calculer soit un nombre de cas attendus de maladies, soit un pourcentage de population susceptible d'être touchée par une pathologie. Conventionnellement, l'EQRM comprend quatre étapes:

\section{a- la caractérisation du danger}

Dans le cadre de l'EDR microbiologique, la caractérisation du danger vise à identifier, dans le milieu considéré, les microorganismes pathogènes potentiellement dangereux pour la santé humaine et les différents effets qui y sont liés. L'un des résultats, de cette analyse du danger, est la prise de décisions quant aux conséquence(s) principalement sanitaires à mesurer dans l'évaluation formelle des risques (Haas et al., 2001).

\section{b- caractérisation de l'exposition}

Le but de l'évaluation des expositions est de déterminer les doses d'agents pathogènes consommées dans l'eau par un individu ou la population. 
L'approche généralement utilisée, pour cette étape, est principalement basée sur une approche épidémiologique consistant en une caractérisation microbiologique des milieux environementaux et des mesures chez le récepteur " humain ». Pour la caractérisation de l'exposition aux microorganismes à partir de l'eau potable, Macler et Regli (1993) suggèrent, une dotation de 2 litres par individu et par jour.

\section{c- la relation dose-effet}

Lors de l'identification des fonctions dose-effet, le choix du modèle est fondamental pour l'estimation du niveau de risque d'infection puisqu'il fournit la probabilité d'infections à partir d'un niveau d'exposition ou, inversement, qu'il estime l'exposition à partir du taux d'attaque dans la population. Jusqu'à maintenant le raisonnement a utilisé divers modèles élaborés sur des données de type dose-effet ou dose-réponse, pour calculer une probabilité d'infections, et un risque annuel ou vie entière associée à tel ou tel milieu. Dans le présent travail, nous avons choisi le modèle Bêta-Poission: il dérive du modèle exponentiel, à la différence que la probabilité de survie du germe n'est pas considérée comme une constante, mais par une distribution de probabilité, et ce pour tenir compte du caractère variable de ce paramètre (Haas 1983b ; Haas et al., 2001). La formulation du modèle est :

$$
P(d)=1-\left(1+\frac{d}{\beta}\right)^{-\alpha} \quad \text { ou } \quad P(d)=1-\left[1+\frac{d}{N_{50}}\left(2^{1 / \alpha}-1\right)\right]^{-\alpha}
$$

$\mathrm{d}:$ dose d'exposition

$\mathrm{N}_{50}$ : dose infectante moyenne

$\alpha$ : paramètre de la fonction de probabilité

\section{d- Caractérisation du risque}

Le processus de la caractérisation de risque combine l'information sur l'exposition et celle sur la relation dose-réponse dans une analyse de probabilités sur l'occurrence des effets défavorables. Ceci peut être fait de deux manières:D'abord, une évaluation simple des points d'exposition (c'est à dire le nombre d'organismes ingérés) peut être combinée avec une évaluation simple des paramètres de la relation dose-effet pour estimer les points de risque. Ceci peut être fait en utilisant une démarche permettant d'obtenir une mesure de tendance centrale, ou d'employer une stratégie extrême visant l'atteinte dune mesure extrême. Une approche alternative, qui est de plus en plus appréciée et utilisée actuellement, est de caractériser la pleine distribution de l'exposition et des rapports dose-réponse, et de combiner ces divers outils en effectuant une distribution aléatoire de risque (Haas et al., 2001). 
Etude épidémiologique des maladies inhérentes à la consommation de l'eau du bassin versant de la Menoua

Le recensement des maladies hydriques a été fait dans les registres de l'hôpital de district de Dschang.

\section{Analyse statistique des données}

Le traitement de l'ensemble de données a été réalisé avec le logiciel XL-STAT. Ces résultats ont été comparés aux normes de l'OMS de la qualité de l'eau destinée à la consommation humaine. Le traitement statistique des donnes épidémiologique a été fait avec le logiciel Epi Info.

\section{Résultats et discussions}

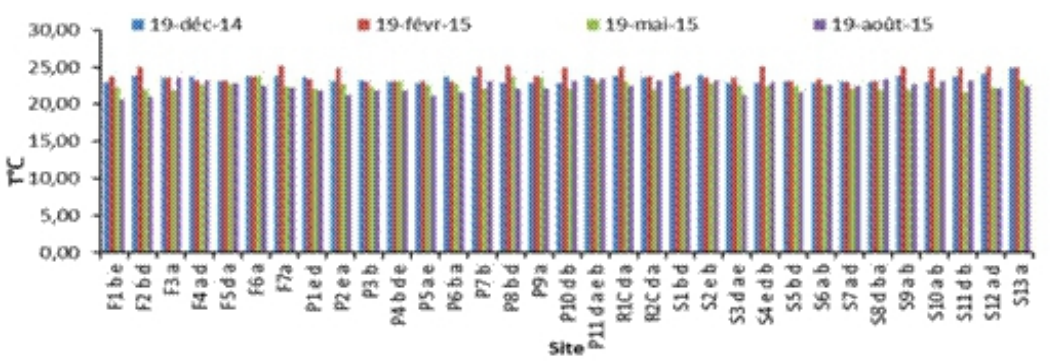

Figure 3: Evolution spatio-temporelle de la température de l'eau du bassin

\section{Paramètres physico-chimiques}

\section{Température}

Les résultats des Figures 3 à 5 montrent des variations spatiotemporelles de la température de l'eau similaires pour toute la période d'étude. L'amplitude de la variation entre les campagnes ne dépasse en aucun cas $25,50{ }^{\circ} \mathrm{C}$. La température maximale $\left(25,20^{\circ} \mathrm{C}\right)$ a été enregistrée pendant la saison sèche (février 2014) au site $\mathrm{P} 8$ et la température minimale $\left(20,70^{\circ} \mathrm{C}\right) \mathrm{a}$ été obtenue pendant la saison pluvieuse (août 2015). Ceci reflète bien l'influence de la température de la localité. Quant aux variations spatiales, aucune différence significative n'a été relevée. Les écarts enregistrés ne sont dûs en fait qu'au décalage horaire journalier entre les différents sites de prélèvement, c'est la raison pour laquelle le profil spatial ne présente pas de différence significative entre les différents sites (Tableau1). La moyenne qui oscille entre $22,40{ }^{\circ} \mathrm{C}$ et $23,90^{\circ} \mathrm{C}$ reste liée aux conditions climatiques qui règnent dans la région (Bouyo, 2002). En effet, la température de l'eau fait partie des facteurs importants contrôlant la quasi-totalité des réactions microbiologiques des milieux aquatiques (Chapman et al., 1996). Elle est acceptable par rapport à la norme de l'OMS. 


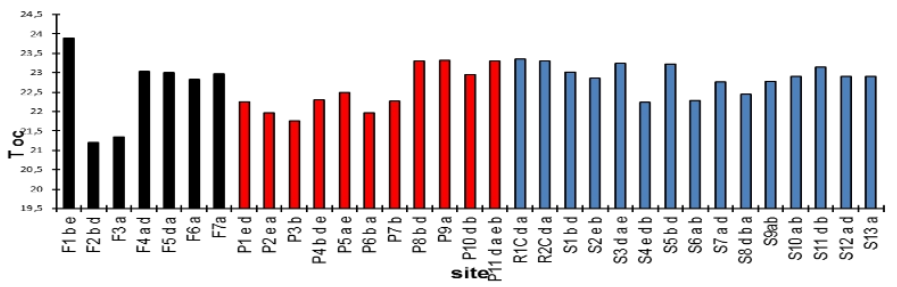

Figure 4: Evolution spatiale des moyennes de la température de l'eau du bassin

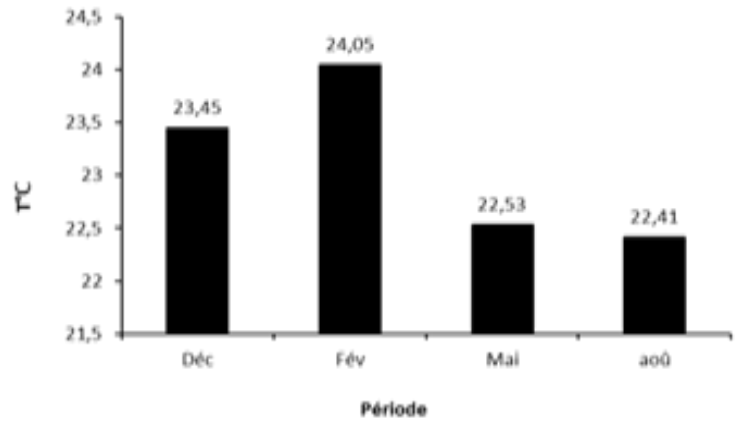

Figure 5: Evolution temporelle de la température de l'eau du bassin

\section{pH (potentiel hydrogène)}

Les valeurs du $\mathrm{pH}$ de l'eau le long du bassin versant de la Menoua (Tableau1) enregistrées au cours de la période de l'étude, varient entre 7,96 enregistrées dans le site RC1 et 4,22 enregistrées dans les sites P4 et P9 au mois de décembre 2014. L'évolution temporelle des moyennes du $\mathrm{pH}$ ne dépasse pas en général une unité. Cependant, comme illustrée par la Figure 6, l'allure des courbes de la variation spatio-temporelle du $\mathrm{pH}$ pour les 33 sites est très variable et ne suit pas une loi régulière. L'évolution spatiale des moyennes du $\mathrm{pH}$ (Figure 7) dans les différents sites étudiés présente un écart remarquable $(1,50)$, la valeur maximale $(6,38)$ étant enregistrée dans le site F3 et la valeur minimale $(5,09)$ enregistrée dans le site P11. L'évolution des moyennes temporelles du $\mathrm{pH}$ (Figure 8) des eaux du bassin versant de la Menoua montrent un $\mathrm{pH}$ variable, mais à tendance acide et ne cadrent pas avec la norme de l'OMS. Cette différence du pH enregistrée est en rapport avec les fluctuations probables des activités agropastorales, les points de décharges ponctuels situés à proximité des points d'eau ou dans les cours d'eau du bassin (Close et al., 1989; Hassoune et al., 2006). Il est à remarquer que les eaux usées ménagères se retrouveraient dans les cours d'eau du bassin sans subir aucun traitement préalable. 


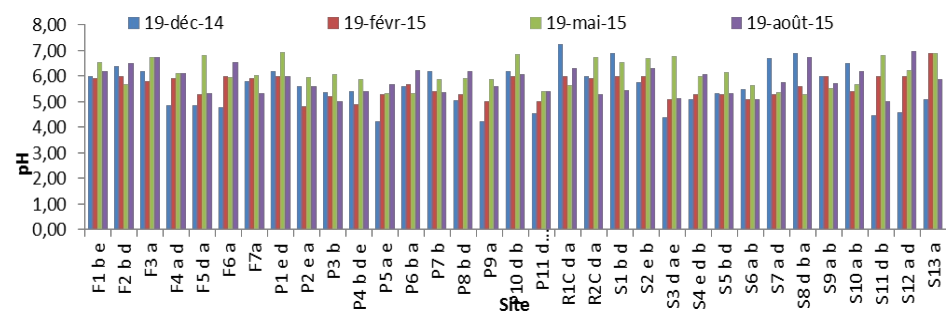

Figure 6: Evolution spatio-temporelle du $\mathrm{pH}$ de l'eau du bassin

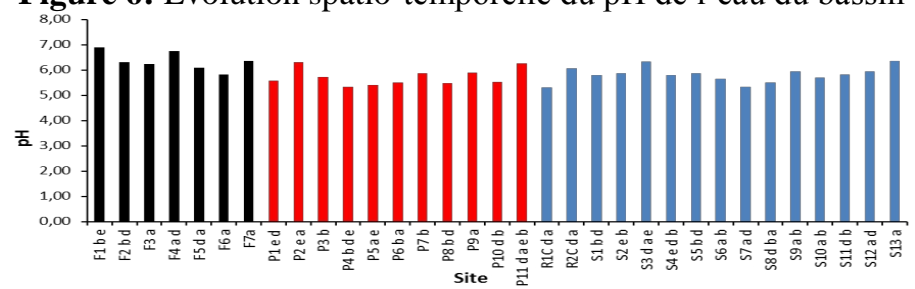

Figure 7: Evolution spatiale des moyennes du pH de l'eau du bassin

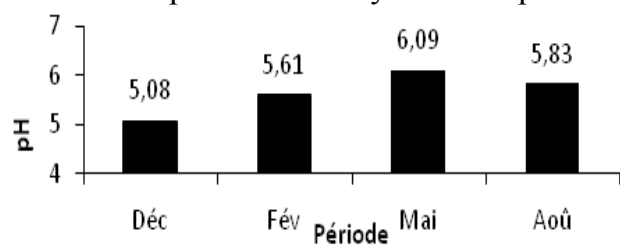

Figure 8: Evolution temporelle du pH de l'eau du bassin

\section{Oxygène dissous (OD)}

Les résultats spatio-temporels de la Figure 9 montrent un faible taux d'Oxygène avec une valeur de $2.00 \mathrm{mg} / \mathrm{l}$ enregistrée dans le site F4. Au mois de février 2015, on note un maximum de l'Oxygène avec une valeur de 9,04 $\mathrm{mg} / \mathrm{l}$ enregistrée dans le site P10. L'évolution des moyennes spatiales (Figure 10) montre une forte valeur $(7,12 \mathrm{mg} / \mathrm{l})$ au niveau du site P10 et une faible valeur $(3,99 \mathrm{mg} / \mathrm{l})$ au niveau du S9. Dans notre étude, l'évolution temporelle des moyennes (Figure 11) de l'Oxygène dissous montre des teneurs plus élevées en saison pluvieuse que celles de la saison sèche. Dans l'ensemble, ces valeurs restent faibles par apport à la norme de (OMS, 2006). Les faible teneurs en Oxygène dissous est dû non seulement à l'absence de végétaux capables de photo synthétiser, au faible contact eau- atmosphère (Abdelaali, 1990), mais aussi aux infiltrations possibles d'eaux usées. L'Oxygène dissous peut se réduire par l'activité des bactéries décomposant la matière organique présente dans le point d'eau (Fekhaoui et al., 1993). 


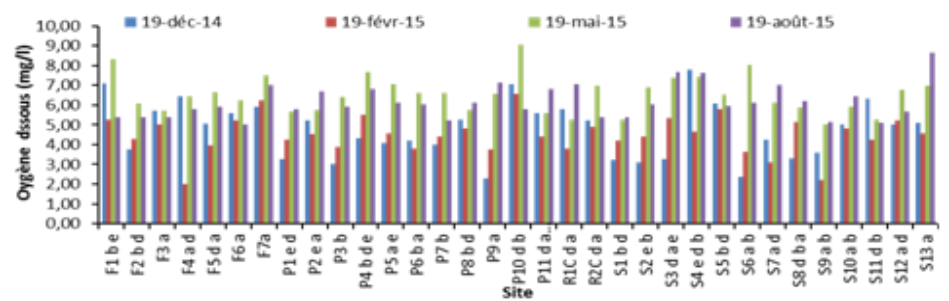

Figure 9: Evolution spatio-temporelle de l'Oxygène dissous de l'eau du bassin

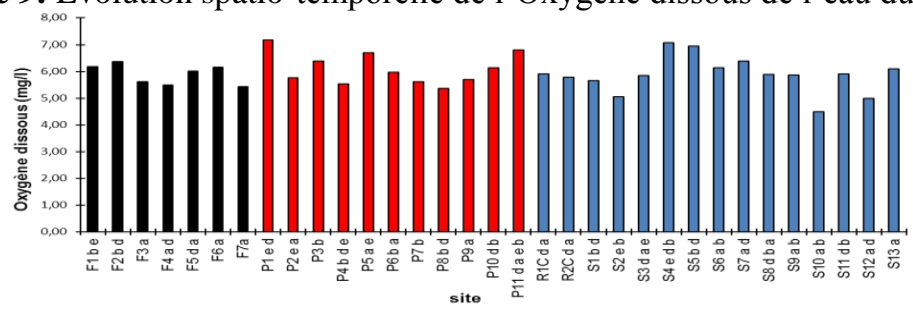

Figure 10: Evolution spatiale des moyennes de l'Oxygène dissous de l'eau du bassin

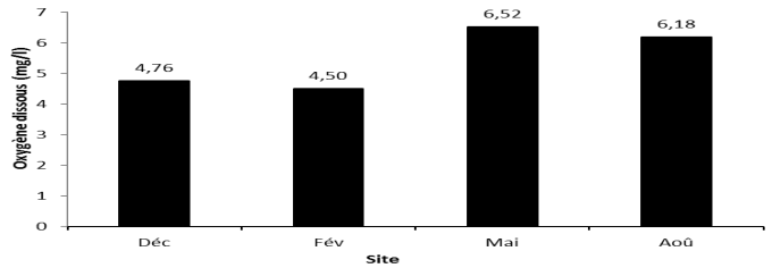

Figure 11: Evolution temporelle de l'Oxygène dissous de l'eau du bassin

\section{Turbidité}

L'évolution saisonnière de la turbidité (Figure 12-14) montre des variations spatiales similaires. La turbidité maximale $(2,01 \mathrm{TFU})$ a été enregistrée au site R2C pendant la saison pluvieuse (août 2015) et la turbidité minimale $(0,27 \mathrm{TFU})$ a été obtenue au site F5 pendant la même saison (mai 2015). Ceci reflète bien la spécificité de chaque site et des activités anthropiques rencontrées. Les valeurs des moyennes spatiales sont maximales $(1,6 \mathrm{TFU})$ au site R2C et minimales $(0,33 \mathrm{TFU})$ au site F2. Le site R2C est un cours d'eau du bassin ouvert aux eaux torrentielles, aux eaux usées domestiques et aux intrants agropastoraux. L'évolution temporelle des moyennes montre que les valeurs de la turbidité sont plus fortes en saison pluvieuse et faibles en saison sèche. ANOVA laisse apparaitre un effet très hautement significatif de la turbidité (Tableau1). Les fortes valeurs de la turbidité d'un point à l'autre de l'eau du bassin versant de la Menoua proviendraient des eaux torrentielles portant avec elles les débris de toutes natures (Rodier et al., 2005). 


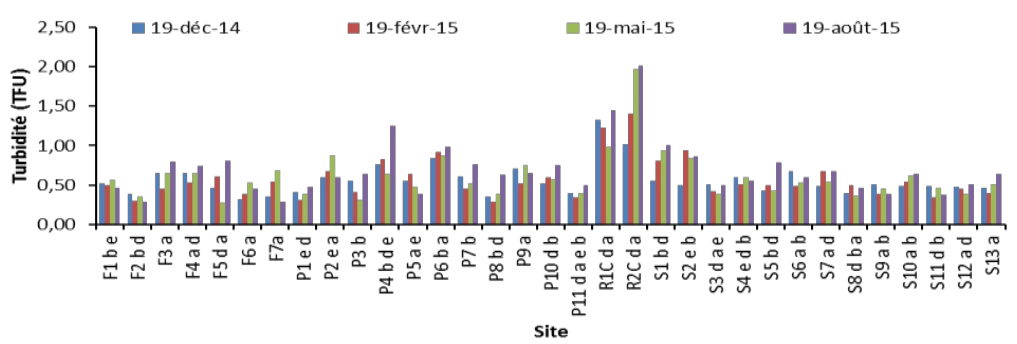

Figure 12: Evolution spatio-temporelle de la turbidité de l'eau du bassin

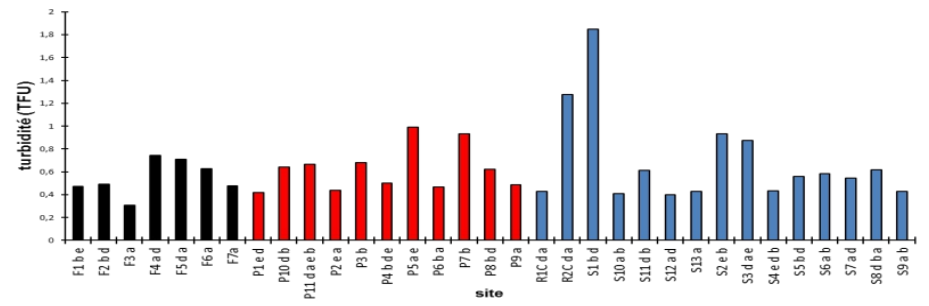

Figure 13: Evolution spatiale des moyennes de la turbidité de l'eau du bassin

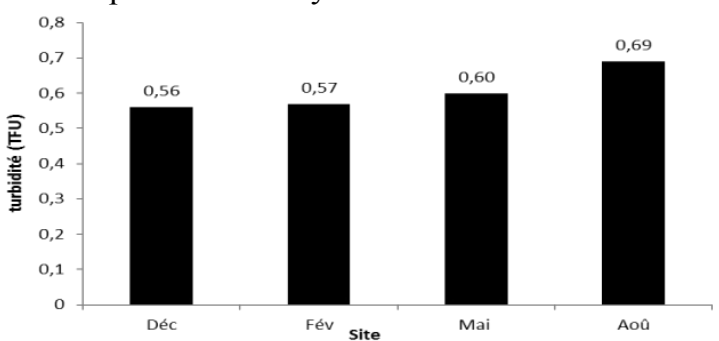

Figure 14: Evolution temporelle de la turbidité de l'eau du bassin

\section{Paramètres Microbiologiques \\ E-colis}

L'évolution spatio-temporelle des E-colis (Figure 15) présente la teneur plus élevée (500UFC/ml) au site P1 en mai 2015. Les autres sites ont des teneurs moins fortes. Ces teneurs oscillent entre $0 \mathrm{UFC} / \mathrm{ml}$ enregistrées aux sites F3, S2, S3 et S4 en février et mai et 300UFC/ml enregistrée au R2C (août). Le profil des moyennes spatiales des E-coli (Figure 16) montre les teneurs oscillant entre $255 \mathrm{UFC} / \mathrm{ml}$ enregistrées au site $\mathrm{P} 1$ et $8 \mathrm{UFC} / \mathrm{ml}$ au site et S6. Ces fluctuations observées dans certains sites seraient probablement dûes à l'utilisation des matières fécales des animaux (Bovins, Ovins et Porcs)comme engrais dans les aires cultivées aux abords des cours d'eau du bassin. La variation des moyennes temporelles de la teneur en E-coli (Figure 17) présente des valeurs variant entre $133,5 \mathrm{UFC} / \mathrm{ml}$ enregistrées en saison pluvieuse et 31,93UFC/ml enregistrées en saison sèche. Les fortes teneurs sont notées en saison pluvieuse par rapport au reste de l'année. La quantité de microorganismes dans ces eaux augmente avec la pluviométrie, bien qu'aucune étude n'ait jusqu'à présent pu établir une loi simple modélisant formellement ces deux paramètres (Ouhmidou et al., 2015). Le lessivage des 
sols par les eaux de ruissellement peut être néanmoins une raison principale pour la mobilisation des biomasses des sols. L'analyse de la variance montre un effet significatif(tableau1). Ces valeurs ne cadrent pas avec la norme de l’organisation mondiale de la santé (OMS, 2006).

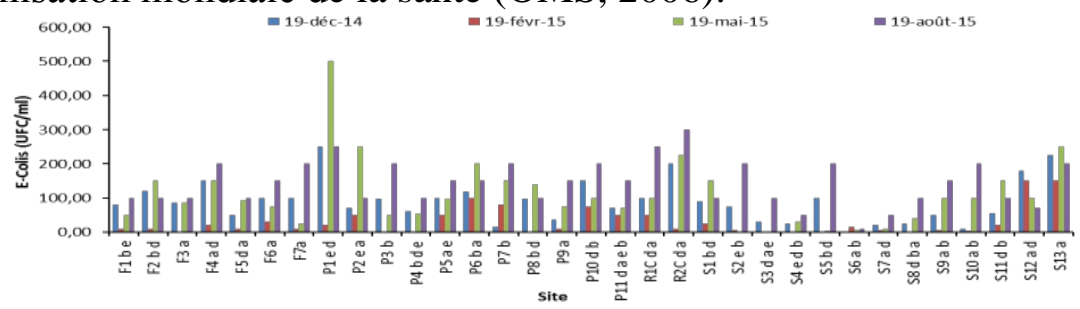

Figure 15:Evolution spatio-temporelle des E-colis de l'eau du bassin

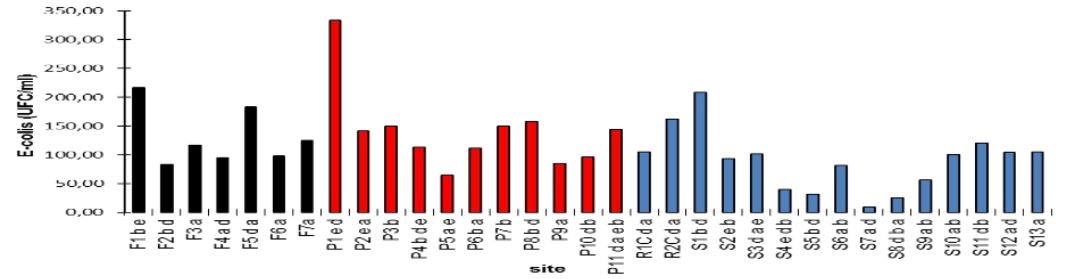

Figure 16:Evolution spatiale des moyennes des E-colis de l'eau du bassin

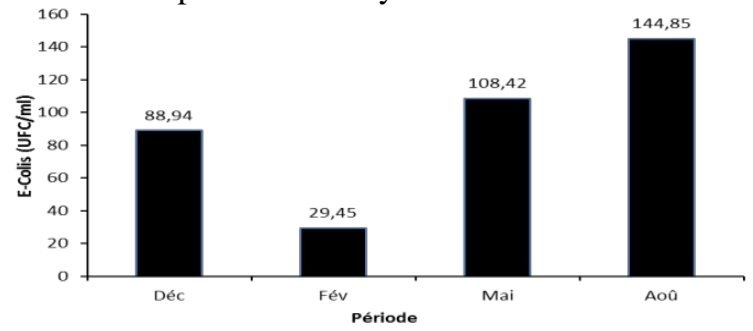

Figure 17:Evolution temporelle des E-colis de l'eau du bassin

\section{Salmonelles}

L'allure des courbes de la variation spatio-temporelle des salmonelles le long du bassin versant de la Menoua (Figure 18) est très variable en août et présente des similitudes dans certains sites. Les valeurs trouvées des salmonelles dans les différents sites oscillent entre 350UFC/ml enregistrées dans le site $\mathrm{S} 11$ et $0 \mathrm{UFC} / \mathrm{ml}$ notée aux sites F3, P3, P4, S2, S3, S4, S7, S8 et $\mathrm{S} 10$ en février 2015. La variation des moyennes spatiales (Figure 19) montre des teneurs oscillant entre 206,25UFC/ml enregistrées au site R1C et 4UFC/ml enregistrées au site S6. Le profil des moyennes temporelles (Figure 20) présente des teneurs plus fortes en saison pluvieuse dans laquelle le maximum est enregistré en août 2015. Les valeurs faibles sont enregistrées en saison sèche pendant laquelle le minimum est noté en février de la même année. Ces différences de teneur s'expliquent par l'élevage des Volailles et des Porcs notés dans certains sites le long du bassin, il en est de même de la matière fécale utilisée comme engrais par les agriculteurs (Maul et al., 1982). Les fortes teneurs enregistrées en saison pluvieuse se justifient par le lessivage des 
lisiers et des fumiers provoqués par les eaux de pluies (Patoine, 2005). L'analyse de la variance ne laisse apparaitre aucun effet significatif des salmonelles dans l'eau des différents sites(tableau1). Ces valeurs ne cadrent point avec la norme de l'organisation mondiale de la santé (OMS, 2006).

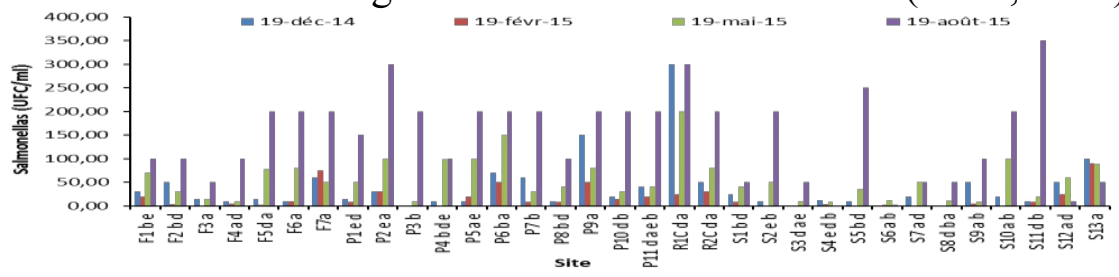

Figure 18:Evolution spatio-temporelle des salmonelles de l'eau du bassin

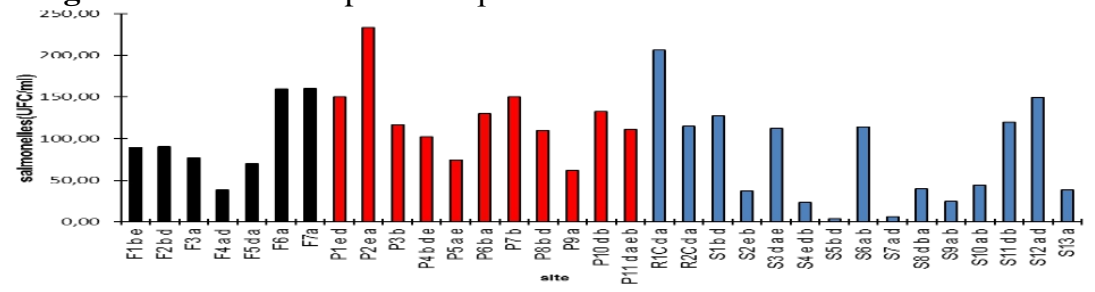

Figure 19: Evolution spatiale des moyennes des salmonelles de l'eau du bassin

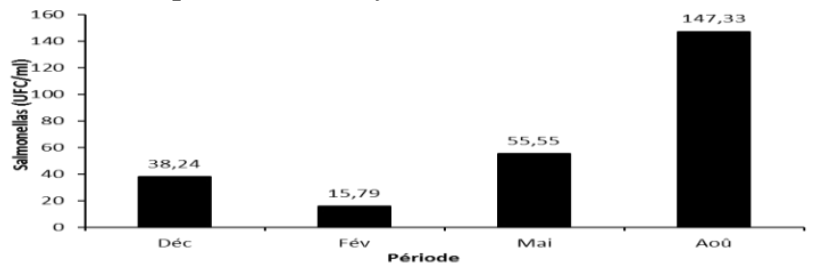

Figure 20: Evolution temporelle des salmonelles de l'eau du bassin

\section{Shigellas}

La variation spatio-temporelle des shigellas (Figure 21) montre des teneurs à faible évolution, sauf dans certains sites où on assiste à une élévation importante au mois de mai et août dont la valeur maximale $(250 \mathrm{UFC} / \mathrm{ml})$ est enregistrée au site R1C. La valeur minimale $(0 \mathrm{UFC} / \mathrm{ml})$ est notée dans la majorité des sites. L'évolution des moyennes spatiales (Figure 22) présente un profil presque similaire à celui du spatio-temporel. Les teneurs maximales (125UFC/ml) sont enregistrées au site R1C et celles minimales (0UFC/ml) au site F3. Le profil des moyennes temporelles (Figure 23) présente une évolution plus importante en saison pluvieuse pendant laquelle la teneur maximale $(39,75 \mathrm{UFC} / \mathrm{ml})$ est enregistrée en août 2015. Les faibles teneurs sont enregistrées en saison sèche avec la valeur minimale $(12,75 \mathrm{UFC} / \mathrm{ml})$ notée en février de la même année. L'évolution des teneurs en shigella dans l'ensemble du bassin sont différents d'un site à un autre et d'une saison à une autre. Ceci pourrait être expliqué par une activité humaine très importante. Les rejets des eaux usées et des déchets solides sans traitement préalable constituent l'origine la plus importante de cette teneur enregistrée dans certains sites (El OualiLalami, 2011). Cette élévation des teneurs en saison pluvieuse s'explique 
par les eaux pluviales qui entrainent dans les cours d'eau les microorganismes (Zamo et al., 2003). L'analyse de la variance montre un effet hautement significatif des shigellas dans l'eau des différents sites (tableau1). Ces valeurs ne cadrent absolument pas avec la norme de l'organisation mondiale de la santé (OMS, 2006).

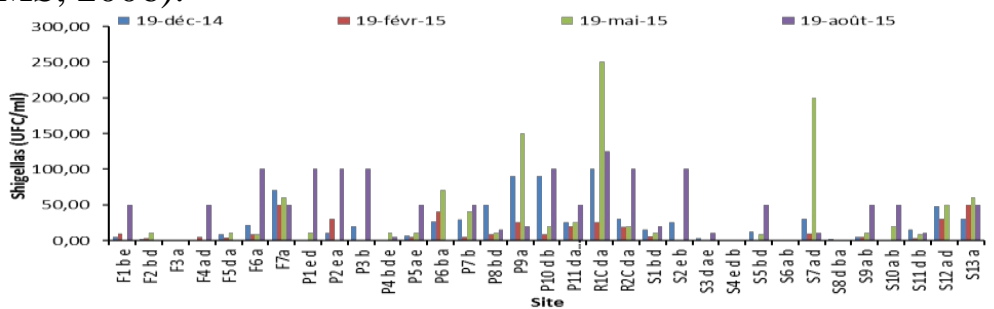

Figure 21: Evolution spatio-temporelle des shigellas de l'eau du bassin

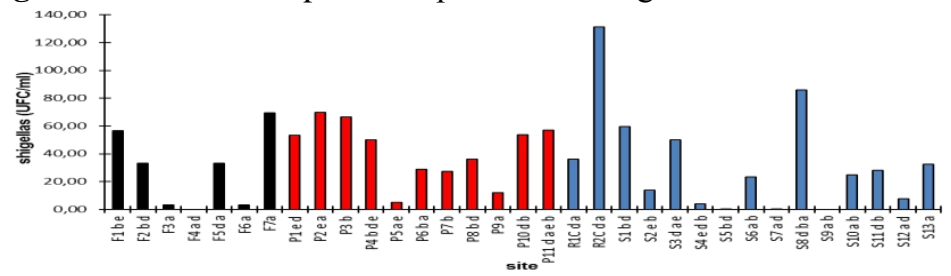

Figure 22: Evolution spatiale des moyennes des shigellas de l'eau du bassin

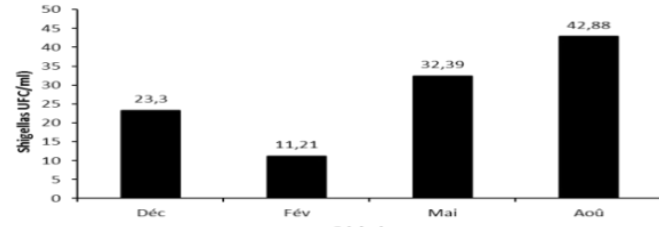

Figure 23: Evolution temporelle des shigellas de l'eau du bassin 
Tableau 1: ANOVA des paramètres physicochimiques et microbiologiques de l'eau des

\begin{tabular}{|c|c|c|c|c|c|c|c|}
\hline \multicolumn{8}{|c|}{ differents sites du bassin } \\
\hline Site & $\mathrm{pH}$ & T oc & $\begin{array}{l}\text { Oxygène } \\
\text { dissous }\end{array}$ & turbidité & E-colis & salmonelles & shigellas \\
\hline $\mathrm{F} 1 \mathrm{~b} \mathrm{e}$ & $6,160^{\mathrm{a}}$ & $22,400^{\mathrm{a}}$ & $6,510^{a}$ & $0,510^{\text {cdefg }}$ & $183,750^{\mathrm{abc}}$ & $90,000^{\mathrm{a}}$ & $42,250^{\mathrm{ab}}$ \\
\hline $\mathrm{F} 2 \mathrm{~b} \mathrm{~d}$ & $6,148^{a}$ & $22,985^{\mathrm{a}}$ & $4,883^{a}$ & $0,330^{\mathrm{g}}$ & $125,000^{\mathrm{abc}}$ & $206,250^{a}$ & $125,000^{a}$ \\
\hline F3 a & $6,370^{\mathrm{a}}$ & $23,175^{\mathrm{a}}$ & $5,448^{\text {a }}$ & $0,635^{\text {cdefg }}$ & $206,250^{a b}$ & $82,250^{a}$ & $47,500^{a b}$ \\
\hline F4 a d & $5,735^{\mathrm{a}}$ & $23,175^{\mathrm{a}}$ & $5,165^{\mathrm{a}}$ & $0,643^{\text {cdefg }}$ & $87,000^{\mathrm{abc}}$ & $89,000^{\mathrm{a}}$ & $60,000^{\mathrm{ab}}$ \\
\hline F5 d a & $5,575^{\mathrm{a}}$ & $22,973^{\mathrm{a}}$ & $5,403^{\mathrm{a}}$ & $0,538^{\text {cdefg }}$ & $125,000^{\mathrm{abc}}$ & $36,250^{\mathrm{a}}$ & $32,000^{\mathrm{ab}}$ \\
\hline F6 a & $5,820^{\mathrm{a}}$ & $23,500^{\mathrm{a}}$ & $5,518^{a}$ & $0,420^{\text {efg }}$ & $111,250^{\mathrm{abc}}$ & $74,500^{\mathrm{a}}$ & $31,000^{\mathrm{ab}}$ \\
\hline F7 a & $5,774^{\mathrm{a}}$ & $23,516^{\mathrm{a}}$ & $6,512^{a}$ & $0,442^{\text {efg }}$ & $131,250^{\mathrm{abc}}$ & $66,250^{\mathrm{a}}$ & $54,500^{\mathrm{ab}}$ \\
\hline P1 e d & $6,288^{\mathrm{a}}$ & $22,750^{\mathrm{a}}$ & $4,743^{\text {a }}$ & $0,393^{\mathrm{fg}}$ & $117,500^{\mathrm{abc}}$ & $115,000^{\mathrm{a}}$ & $35,000^{\mathrm{ab}}$ \\
\hline $\mathrm{P} 2 \mathrm{e}$ a & $5,503^{\mathrm{a}}$ & $23,025^{\mathrm{a}}$ & $5,553^{\text {a }}$ & $0,683^{\text {cdefg }}$ & $67,500^{a b c}$ & $120,000^{\mathrm{a}}$ & $71,250^{\mathrm{ab}}$ \\
\hline P3 b & $5,423^{\text {a }}$ & $22,625^{\mathrm{a}}$ & $4,805^{\mathrm{a}}$ & $0,478^{\operatorname{defg}}$ & $142,000^{\mathrm{abc}}$ & $117,500^{\mathrm{a}}$ & $34,000^{\mathrm{ab}}$ \\
\hline P4 bd e & $5,405^{a}$ & $22,775^{a}$ & $6,070^{a}$ & $0,870^{\text {bcd }}$ & $87,250^{\mathrm{abc}}$ & $52,500^{\mathrm{a}}$ & $30,000^{\mathrm{ab}}$ \\
\hline P5 a e & $5,135^{\mathrm{a}}$ & $22,423^{\mathrm{a}}$ & $5,458^{a}$ & $0,510^{\text {cdefg }}$ & $85,000^{a b c}$ & $75,000^{\mathrm{a}}$ & $30,000^{\mathrm{ab}}$ \\
\hline P6 b a & $5,720^{a}$ & $22,788^{a}$ & $5,158^{a}$ & $0,900^{\mathrm{bc}}$ & $91,250^{\mathrm{abc}}$ & $30,750^{\mathrm{a}}$ & $12,750^{b}$ \\
\hline P7 b & $5,703^{a}$ & $23,468^{a}$ & $5,060^{\text {a }}$ & $0,585^{\text {cdefg }}$ & $78,250^{a b c}$ & $80,000^{a}$ & $17,500^{b}$ \\
\hline P8 b d & $5,620^{\mathrm{a}}$ & $23,495^{\mathrm{a}}$ & $5,480^{a}$ & $0,415^{\text {efg }}$ & $81,250^{a b c}$ & $97,000^{\mathrm{a}}$ & $9,000^{b}$ \\
\hline P9 a & $5,175^{\mathrm{a}}$ & $23,143^{\mathrm{a}}$ & $4,933^{\text {a }}$ & $0,658^{\text {cdefg }}$ & 88,750 abc & $75,000^{\mathrm{a}}$ & $34,500^{\mathrm{ab}}$ \\
\hline P10 a b & $6,285^{a}$ & $23,250^{\mathrm{a}}$ & $7,118^{a}$ & $0,610^{\text {cdefg }}$ & $85,000^{\mathrm{abc}}$ & $39,500^{\mathrm{a}}$ & $20,750^{b}$ \\
\hline $\mathrm{P} 11 \mathrm{~d}$ a e b & $5,095^{\mathrm{a}}$ & $23,450^{\mathrm{a}}$ & $5,605^{\mathrm{a}}$ & $0,408^{\mathrm{fg}}$ & $255,000^{a}$ & $55,750^{\mathrm{a}}$ & $27,500^{a b}$ \\
\hline $\mathrm{R} 2 \mathrm{C} \mathrm{d} \mathrm{a}$ & $5,983^{\mathrm{a}}$ & $23,125^{\mathrm{a}}$ & $5,620^{\mathrm{a}}$ & $1,600^{\mathrm{a}}$ & $76,000^{\mathrm{abc}}$ & $74,000^{\mathrm{a}}$ & $17,750^{b}$ \\
\hline $\mathrm{R} 1 \mathrm{C}$ da & $6,305^{\mathrm{a}}$ & $23,625^{\mathrm{a}}$ & $5,475^{a}$ & $1,243^{a b}$ & $60,000^{\mathrm{abc}}$ & $55,000^{\mathrm{a}}$ & $16,000^{b}$ \\
\hline $\mathrm{S} 1 \mathrm{~b} \mathrm{~d}$ & $6,225^{\mathrm{a}}$ & $23,243^{\mathrm{a}}$ & $4,515^{\mathrm{a}}$ & $0,823^{\text {cde }}$ & $26,250^{\mathrm{bc}}$ & $5,750^{\mathrm{a}}$ & $0,250^{\mathrm{b}}$ \\
\hline $\mathrm{S} 2 \mathrm{e} \mathrm{b}$ & $6,195^{\mathrm{a}}$ & $23,430^{\mathrm{a}}$ & $5,103^{\mathrm{a}}$ & $0,783^{\mathrm{cdef}}$ & $21,250^{b c}$ & $30,000^{\mathrm{a}}$ & $62,250^{\mathrm{ab}}$ \\
\hline $\mathrm{S} 3 \mathrm{~d}$ a e & $5,360^{\mathrm{a}}$ & $22,575^{\mathrm{a}}$ & $5,913^{a}$ & $0,453^{\text {efg }}$ & $99,250^{\mathrm{abc}}$ & $82,500^{\mathrm{a}}$ & $18,000^{b}$ \\
\hline $\mathrm{S} 4 \mathrm{e} \mathrm{d} \mathrm{b}$ & $5,605^{\mathrm{a}}$ & $23,378^{a}$ & $6,875^{a}$ & $0,560^{\text {cdefg }}$ & $54,000^{\mathrm{bc}}$ & $52,000^{\mathrm{a}}$ & $3,750^{\mathrm{b}}$ \\
\hline S5 b d & $5,818^{a}$ & $23,425^{a}$ & $3,992^{\text {a }}$ & $0,432^{\text {efg }}$ & $70,000^{\mathrm{abc}}$ & $65,000^{\mathrm{a}}$ & $31,250^{\mathrm{ab}}$ \\
\hline S6 a d & $5,338^{\mathrm{a}}$ & $22,863^{\mathrm{a}}$ & $5,033^{\text {a }}$ & $0,568^{\text {cdefg }}$ & $63,500^{\mathrm{abc}}$ & $73,750^{\mathrm{a}}$ & $5,500^{\mathrm{b}}$ \\
\hline S7 a d & $5,780^{\mathrm{a}}$ & $22,675^{\mathrm{a}}$ & $5,125^{\mathrm{a}}$ & $0,590^{\text {cdefg }}$ & $130,000^{\mathrm{abc}}$ & $31,250^{\mathrm{a}}$ & $13,750^{\mathrm{b}}$ \\
\hline $\mathrm{S} 8 \mathrm{~d} \mathrm{~b} \mathrm{a}$ & $6,140^{\mathrm{a}}$ & $22,875^{\mathrm{a}}$ & $5,128^{a}$ & $0,430^{\text {efg }}$ & $76,250^{\mathrm{abc}}$ & $40,750^{\mathrm{a}}$ & $17,500^{b}$ \\
\hline S9 a b & $5,735^{\mathrm{a}}$ & $23,175^{a}$ & $5,165^{a}$ & $0,643^{\text {cdefg }}$ & $67,500^{\mathrm{abc}}$ & $20,000^{a}$ & $0,000^{\mathrm{b}}$ \\
\hline $\mathrm{S} 10 \mathrm{a} b$ & $5,940^{\mathrm{a}}$ & $23,340^{\mathrm{a}}$ & $5,548^{\text {a }}$ & $0,570^{\text {cdefg }}$ & $95,000^{\mathrm{abc}}$ & $45,750^{\mathrm{a}}$ & $3,750^{\mathrm{b}}$ \\
\hline $\mathrm{S} 11 \mathrm{~d} \mathrm{~b}$ & $5,580^{a}$ & $23,350^{\mathrm{a}}$ & $5,225^{\text {a }}$ & $0,413^{\text {efg }}$ & $8,000^{\mathrm{c}}$ & $4,000^{\mathrm{a}}$ & $0,250^{\mathrm{b}}$ \\
\hline $\mathrm{S} 12$ a d & $5,953^{\mathrm{a}}$ & $23,388^{a}$ & $5,665^{\mathrm{a}}$ & $0,453^{\text {efg }}$ & $32,750^{b c}$ & $14,750^{\mathrm{a}}$ & $3,250^{\mathrm{b}}$ \\
\hline $\mathrm{S} 13 \mathrm{a}$ & $6,188^{a}$ & $23,913^{\mathrm{a}}$ & $6,323^{a}$ & $0,503^{\text {cdefg }}$ & $41,500^{b c}$ & $15,250^{\mathrm{a}}$ & $0,500^{\mathrm{b}}$ \\
\hline $\operatorname{Pr}>\mathrm{F}$ & 0,060 & 0,968 & 0,518 & $<0,0001$ & 0,003 & 0,284 & 0,002 \\
\hline DF & Non & Non & Non & Oui & Oui & Non & Oui \\
\hline
\end{tabular}

\section{Evaluation du niveau de risque microbiologique lié à la consommation de l'eau du bassin versant de la Menoua}

Afin de déterminer le niveau de risque microbiologique lié à l'ingestion de l'eau du bassin, les microorganismes retenus sont : E-coli, salmonelle et schigella, ceci à cause de leur présence en quantité évaluable, de la disponibilité de leurs valeurs de dose infectante moyenne $\left(\mathrm{N}_{50}\right)$, du paramètre de la fonction de distribution ( $\alpha$ )et de la connaissance de leurs effets épidémiologiques sur l'homme. Notre étude a été portée sur la voie orale et ne concerne que les expositions chroniques (tableau 2). Ainsi, Haas et al., (2001) recommandent pour le Salmonelle $\mathrm{N}_{50}=3,60 \times 10^{6}$ et $\alpha=0,1086$, Haas et al., (1999) préconisent pour Shigella $\mathrm{N}_{50}=1120$ et $\alpha=0,2100$ et pour $\mathrm{E}-$ coli $\mathrm{N}_{50}=$ $8,60 \times 10^{7}$ et $\alpha=0,1778$. 
Tableau 2: Niveau de risque microbiologique lié à la consommation de l'eau du bassin

\begin{tabular}{lcccc}
\hline Microorganismes & $\mathrm{C}$ & $\mathrm{N}_{50}$ & $\alpha$ & Bêta-Poisson $\mathrm{P}(\mathrm{d})$ \\
Salmonelles & 64,93 & $3,60 \times 10^{6}$ & 0,1086 & $1,15 \times 10^{-3}$ \\
Shigellas & 28,23 & 1120 & 0,2100 & $10^{-1}$ \\
E- colis & 88,99 & $8,60 \times 10^{7}$ & 0,1778 & $8,89 \times 10^{-6}$ \\
\hline
\end{tabular}

Tableau 3: Pourcentage des maladies hydriques des populations du bassin

\begin{tabular}{lccc}
\hline Maladies/Germes & sexe & $\%$ & $\%$ totale \\
Typhoïde/Salmonelle & $\mathrm{M}$ & 36,90 & 78,78 \\
& $\mathrm{~F}$ & 65,17 & \\
Gastroentérique/E-coli & $\mathrm{M}$ & 44,22 & 33,02 \\
& $\mathrm{~F}$ & 63,77 & \\
Dysenterie bactérienne/Schigella & $\mathrm{M}$ & 41,13 & 24,61 \\
& $\mathrm{~F}$ & 58,87 & \\
\hline
\end{tabular}

L'UFC/mldes microorganismes présents dans l'eau du bassin est supérieur à 1 , ce qui est au-delà de la norme prescrite par l'OMS. Ces résultats confirment l'existence de dangers pour la santé de la population qui consomme cette eau, et donc la nécessité d'une estimation du niveau des risques sanitaires pour la population concernée et des maladies hydriques récurrentes (tableau 3). Cette Caractérisation des risques infectieux se présente comme suit :

- Le niveau de risque d'infection des populations pour le cas des salmonelles a donné un résultat de $1,15 \times 10^{-3}$ infection par an et par personne. Par ailleurs, $10^{-4}$ infection par an et par personne a souvent été avancée comme le niveau de risques liés à la consommation de l'eau. Ce niveau maximal est remis en cause et considéré comme irréaliste pour certains (Haas, 1996). En effet, des estimations de Centres for Disease Control and Prevention indique que le nombre total de troubles pathologiques liés à l'eau potable serait de plusieurs millions de cas par an aux Etats-Unis, soit un taux annuel de $1 \%$. Haas (1996) note que le critère de $10^{-4}$ paraît alors inapproprié et hors portée, et considère un objectif de $10^{-3}$ infection par an et par personne (voire plus élevé) comme plus adapté. Dans un contexte tropical où la température contribue favorablement au développement et à la croissance des germes pathogènes, $1,15 \times 10^{-3}$ infection par an et par personne indique une très forte contamination de l'eau par des activités anthropiques génératrices des polluants et le haut niveau de risque bactériologique, auquel est exposée la population cible (Evens, 2003). Ce résultat confirme les études épidémiologiques des maladies hydriques, que nous avons menées à l'hôpital de district de Dschang où le pourcentage de la fièvre typhoïde est le plus élevé des maladies hydriques: 78,78\% de la population souffre de la fièvre typhoïde et les femmes sont plus exposées à cette maladie que les hommes avec $65,17 \%$ 
contre 36,90\% (enregistrés en 2015). La fièvre typhoïde est une maladie courante dans les pays les moins industrialisés, principalement du fait de leurs problèmes d'insécurité concernant l'eau. Le résultat de $1,15 \times 10^{-3}$ infection par an et par personne montre combien de fois l'eau est le principal vecteur de cet agent pathogène. Après ingestion, les salmonelles colonisent la muqueuse de l'iléon terminal, au niveau des entérocytes et des plaques de Peyer, puis diffusent par voie lymphatique et sanguine (Goita, 2013).

- Le niveau de risque d'infection lié à E-coli par ingestion de l'eau du bassin calculé dans notre étude est de $8,89 \times 10^{-6}$ infection par an et par personne. Ce risque montre que l'ingestion de l'eau du bassin est potentiel dans la survenue des gastroentériques (Gilles, 2007). Les études épidémiologiques que nous avons menées à l'hôpital de district de Dschang au cours de l'année 2015 montrent que 33,02\% de la population souffrent des gastroentériques et que les femmes sont les couches les plus vulnérables $(63,77 \%)$ contre $(44,22 \%)$ pour les hommes. Ce pourcentage corrobore avec le niveau de risques d'infection estimé et montre encore une fois de plus l'importance que joue l'eau comme principale source d'infection d'E-coli (Gouali, 2013).L'infection d'E-coli survient généralement après ingestion de l'eau polluée ou après un contact avec des personnes ou des animaux porteurs de la bactérie (Aumaître, 2004). E-coli, capable de résister à l'acidité gastrique, passe par l'intestin grêle et colonisent le côlon, en formant les lésions d'attachement et d'effacement se manifestant cliniquement par l'apparition de diarrhées aqueuses (Malika, 2013).

- Le niveau de risques d'infection au shigella par ingestion de l'eau du bassin calculé dans notre étude est de $10^{-1}$ infection par an et par personne. Ce risque est aussi très élevé pour la consommation de cette eau. Les études épidémiologiques que nous avons menées à l'hôpital de district de Dschang au cours de l'année 2015 présente $24,61 \%$ des personnes infectées par shigella et les femmes occupent toujours le premier rang avec un pourcentage de 58,87 par rapport aux hommes dont le pourcentage est de 41,13. Le Shigella provoque des infections digestives de gravité variable. Après pénétration par ingestion de l'eau polluée, les shigellas envahissent la muqueuse de la partie terminale de l'iléon et du gros intestin. La dysenterie bactérienne (shigellose) est une maladie dramatique dans les pays en voie de développement et constitue par conséquent un véritable défit de santé publique (Pierre, 2007).

Dans l'ensemble, la consommation de l'eau du bassin versant de la Menoua sur le plan microbien présente un impact réel sur la santé des consommateurs de la localité de Dschang pour la salmonelle, le shigella, le Ecoli (INVS, 2000). Les études épidémiologiques menées à l'hôpital de district de Dschang, montrent que78,78\% de la population sont exposées à la fièvre typhoïde, 33,02\% aux gastroentériques et 24,61\% à la dysenterie bactérienne 
et que les femmes sont plus exposées. Ces pourcentages corroborent avec le niveau de risques d'infection estimé dû à l'ingestion de l'eau.

\section{Conclusion}

L'étude des paramètres physicochimiques et microbiologiques de l'eau du bassin versant de la Menoua à travers une dynamique spatiotemporelle a mis en évidence une forte pollution microbienne et physicochimique de l'eau vis-à-vis des caractéristiques des apports anthropiques. Cette pollution est appréciable par le pH acide, la faible Oxygénation de l'eau, une forte turbidité de l'eau et des teneurs très importantes en microorganismes traduites par Ecoli, salmonelle et schigella. Par rapport à la Norme de l'OMS de qualité pour l'eau destinée à la consommation humaine, elle n'est pas acceptable. L'estimation du niveau de risque d'infection des populations inhérente à la consommation de cette eau montre qu'elle présente un haut risque microbiologique et que le niveau de risque pour Salmonelle est de $1,15 \times 10^{-3}$ infections par an et par personne, pour E- coli de 8,89x $10^{-6}$ infections par an et par personne et pour Schigella de $10^{-1}$ infections par an et par personne. L'étude épidémiologique présente la typhoïde comme la maladie hydrique la plus récurrente dans le bassin. Enfin, les résultats obtenus à partir de cette étude et dans cette dynamique participative aideront les décideurs à mieux se prononcer sur la gestion des ressources en eau dans ce bassin par le traitement des rejets et l'implantation d'une décharge contrôlée des déchets ménagers afin de pallier les risques de pollution de ces eaux et de préservation de la santé des populations.

\section{References:}

1. APHA (American Public Health Association), American Water Works Association (AWWA) and Water Pollution Control Federation (WPCF).(1981). -Standard Methods for the Examination of Water and Wastewater.15 ${ }^{\text {th }}$ ed APHA. Washington, DC 20005.

2. Chapman D., Kimstach V. (1996). -Selection of Water Quality Variables. Water Quality Assessments: a Guide to the Use of Biota, Sediments and Water in Environment Monitoring. (2nd ed); Chapman edition, E and FN Spon: London; p. 59-126.

3. Close ME, Hodgson LR, Todgre G. (1989).-Field evaluation of fluorescent whiteningagents and sodium tripolyphosphateas indicator of septic tank contamination indomestic wells. New Zeal. J. Marine Fresh. Res., 23: 563-568.

4. Djeuda H.B., Tanawa E., Siakeu J., Ngnikam E. (1998).- Contraintes sociales liées à la mise en place des périmètres de protection des ressources en eau dans les zones périurbaines et les petits centres des pays en développement. Communication présentée au IIème 
Symposium International en gestions et technologies appropriées pour l'eau aux petits ensembles habités. Barcelone (Espagne). Du 13 au 15octobre 1998. $11 \mathrm{p}$

5. Durand, J.R., Leveque, C. (1980). -Flore et Faune aquatique de l'Afrique Sahelo Soudanienne. Tome I. 2 ditions de l'Office de la recherche Scientifique d'Outre-Mer (O.R.S.T.O.M.) Collection Initiations-Documentations. Technique $\mathrm{n}^{\circ} 44$. Paris

6. Dutertre J., Huet M., Gateff C., Durand B. (1972). -Le choléra au Cameroun. Med Trop 1972 ; 32 : 607-24.

7. EL Kettani, Azzouzi E. M. (2006).-Prévalence des helminthes au sein d'une population rurale utilisant les eaux usées à des fins agricoles à Settat (Maroc). Environnement Risques et Santé, 2 : 99-106.

8. EL OualiLalami, A. (2011). -Pollution des eaux de puits de certains quartiers de la ville de Fès, Maroc, Rev. Microbiol. Ind. San et Environn. 5 (1) (2011) 37-68.

9. ERA-CAMEROUN. (2001). -Projet d'assainissement autonome du quartier Melen 4 Yaoundé. Rapport d'enquête d'identification des latrines et des points d'eau. 52p

10. Evens E. (2003). Evaluation des risques sanitaires et éco toxicologiques lies aux effluents hospitaliers. Thèse pour obtenir le grade de Docteur de l'Institut National des Sciences Appliquées de Lyon Sciences et Techniques du Déchet Ecole Doctorale de Chimie de Lyon, 172-179.

11. Fekhaoui M., Pattee E. (1993). -Impact de la ville de Fés sur l'oued Sebou : étude physicochimique. Bull. Ins. Sci.( Rabat), 17, 112.

12. Haas C.N, einsenberg J.N.S.(2001) -Risk assessment. In.: Fewtrell L., Bartram W., Bartram J. (eds)Water Quality - Guidelines, standards and health: Assessement of Risk Management for water-related infectious disease. World Health Organization, Gevena,. Available on.:www.who.int/water_sanitation_health/Documents/IWA/iwabookc hap8.htm, 22/06/2016.

13. Haas C.N., Rose J.B., GerbaC.P.(1999). -Quantitative Microbial Risk Assessment. Wiley, New York,449 p.

14. haas, C.N.(1983b) -Estimation of risk due to low doses of microorganisms: A comparison of alternative methodologies. American Journal of Epidemiology, vol.118, 4:573-582.

15. Hass C.N.(1996). -Acceptable microbial risk. J. Am. Water Works Ass., Vol. $88, \mathrm{n}^{\circ} 12$.

16. Hassoune E. M., Bouzidi A., Koulali Y. et Hadarbach D. (2006). Effets des rejets liquides domestiques et industriels sur la qualité des eaux souterraines au nord de la ville de Settat (Maroc). Bulletin de l'Institut Scientifique, Rabat, section Sciences de la Vie, n²8, 61-71. 
17. Hassoune EM, Bouzidi A, Koulali Y, Hadarbach D. (2006). - Effets des rejets liquides domestiques et industriels sur la qualité des eaux souterraines au nord de la ville de Settat (Maroc). Bulletin de l'Institut Scientifique, Rabat, section Sciences de la Vie, 28: 61-71.

18. INS (Institut National de la Statistique). (2010). -Annuaire Statistique $d u$ Cameroun 2010.INS: Yaoundé-Cameroun.

19. IVS (institut de veille sanitaire). (2000). -Guide pour l'analyse du volet sanitaire des études d'impact. Institut de Veille Sanitaire, SaintMaurice Cédex, 49 p., ISBN 2-11-09 14 82-3.

20. Kamgho T.B.M. (2010).-L'accès à l'eau potable et à l'assainissement au Cameroun : situation actuelle, contraintes, enjeux et défis pour l'atteinte de l'OMD 7, 8-11

21. Kengni L., Tematio P., Filali K., Tepoule J., Tsafack E. I., Mboumi T. L. Mounier. (2012).-Pollution des eaux superficielles et des nappes en milieu urbain : cas de la zone industrielle de Douala-Bassa (Cameroun).Int. J. Biol. Chem. Sci. 3(3): 441-447, 2009.

22. Kouam G.R.,RosillonF., Nono A., Nzeukou A., Mpakam H.G. (2011).Les maladies hydriques à l'épreuve de la gestion des ressources en eau dans une zone urbaine tropicale : cas de Yaoundé (Cameroun). Eur. Journ. of WaterQuality.Paris, France. 15P. DOI 10.1051/water/2011004.

23. Kuitcha D., Kamgang V., Sigha L., Lienou G., Ekodeck G.E. (2008).Water supply, sanitation and health risk in Yaounde, Cameroon. African Journal of Environmental Science and Technology, Vol. 2 (11).PP. 379-386.

24. Laferriere M., Minville J.J., Lavoie J., Payment P. (1996). -L'industrie porcine et les risques reliés à la santé humaine. Bull. Information Santé Environnement., Québec, 7(2): 1-4.

25. lewa (2005). -Evaluation de la qualité des eaux de boisson et de ménage dans le département de la Vina. Mémoire de diplôme d'études approfondies. Université de Yaoundé. $59 \mathrm{p}$.

26. Lewa S., Ombolo A., Loura B. B. (2014).-Qualité et pollution des eaux d'un hydrosystème littoral tropical : cas Du système lagunaire de lomé, togo European Scientific Journal May 2015 edition vol.11, No.15 ISSN: 1857 - 7881 (Print) e - ISSN 1857- 7431

27. Macler B.A., Regli S. (1993)-Use of microbial risk assessment in setting United States drinking water standards. International Journal of Food Microbiology,vol. 18, 4:245-256.

28. Maul A., Dollard M.A., Block J.C. (1982).-Etude de l'hétérogénéité spatiotemporelle des bactéries coliformes en rivière. Journal Français d'Hydrologie, 13, Fasc. 2, no 38, (1982) 141-156.

29. Mc Kinney M.L. (2002). -Urbaization, biodiversity and conservation. 
Biosci., 52, 883-890.

30. Mpakam H. G., Kouam G.R., Tamo T, Maire E.,Boeglin J.L., Ekodeck G.E.,Dupre B. (2009). -Etude des facteurs de pollution des ressources en eau en milieu urbain : cas de Bafoussam (Ouest-Cameroun). Actes $d u$ colloque international sur le thème " changements climatiques et évaluation environnementale »,Niamey (Niger) du 26 au 29 Mai 2009. 27P.

31. Ngnikam E., Tanawa E., Noumba I., Mougoue B., Tabue J. G.,Feumba R.,MeliJ. (2011).-Eau et Santé : Réconcilier l'eau, l'assainissement et la santé par l'approche ECOSANTE. Edition de l'UTBM (Université de Technologie de Belfort Montbéliard). Collection Chantier. 329p.

32. Nkhuwa D.C.W.(2003). -Human activities and threats of chronics epidemics in a fragile geologic environment. Physics and Chemistry of the Earth, 28 : 1139-1145.

33. OMS.(2006). -Directives de qualité pour l'eau de boisson, troisième édition. Recommandations, Organisation mondiale de la Santé, Genève, $78 \mathrm{p}$.

34. Patoine M. (2011). -Influence de la densité animale sur la concentration des coliformes fécaux dans les cours d'eau du Québec méridional, Canada. Revue des sciences de l'eau, 24(4), (2011) 421-435.

35. Rodier JC. et al. (1996). -L'analyse de l'eau naturelle, eaux résiduaires, eau de mer, $8^{\text {ème }}$ Edition, Dénod, Paris, $1383 \mathrm{p}$

36. Rodier JC. et al. (2009). -L'analyse de l'eau: l'eau naturelle eaux résiduaires, eau de mer, $9^{\text {ème }}$ Edition, Dunod, Paris. $1579 \mathrm{p}$

37. Sloat S.,Ziel C. (1992). -The Use of indicator organisms to Assess Public Water Safety. Technical Information Series-Booklet $\mathrm{N}^{\circ}$ 13.Hach Technical Center for Applied Analytical Chemistry, HACH Company. Loveland, Colorado U.S.A.

38. UN-HABITAT (2005). -Municipalité de Dschang: une ville au passé glorieux face aux nouveaux défis.

39. Wethe J., Radoux M.,Tanawa E. (2003).-Assainissement des eaux usées et risques socio-sanitaires et environnementaux en zone d'habitat planifié de Yaoundé (Cameroun). VertigO. La revue électronique en Sciences de l'Environnement, Vol. 4, $\mathrm{N}^{\circ} 1$. 18P.

40. Zamo A.C., Belghiti D., Lyagoubi M. (2003). - Pollution par les eaux usées: Analyse parasitologique des eaux usées brutes et des cultures maraîchères, étude de la viabilité des helminthes et évaluation du risque sanitaire humain dans la région du Gharb (Kénitra) Journal Européen d'hydrologie, Tome 34, fasc 2, 77-90 